\title{
Parametric Probability Distribution
}

National Cancer Institute

\section{Source}

National Cancer Institute. Parametric Probability Distribution. NCI Thesaurus. Code C82844.

A generic data type to specify an uncertain value of an ordered data type using a parametric method (i.e., a distribution function and its parameters are specified). In addition to the specific parameters of the distribution, a mean and standard deviation are always specified. 